\title{
Temperature Measurement System of Steel Strips in a Continuous Annealing Furnace*
}

\section{By Tohru IUCHI,** Jiro OHNO ${ }^{* *}$ and Riichiro KUSAKA ${ }^{* *}$}

\begin{abstract}
Synopsis
In order to measure the temperature of steel strips in a continuous annealing furnace using inert or reducing gases, following items were studied:

(1) The emissivity characteristics of steel strips. These emissivities depended on the specification and temperature of strips as well as the detecting wavelength.

(2) Shielding method of stray radiation energy from furnace walls or heat sources. The stray energy was found to depend on the shape and the surface condition of the shielding flange, distance between the strip and flange, detecting wavelength and temperatures of the furnace wall and the steel strip.

On the basis of these results, a system for measuring strip temperatures was designed and was constructed from a radiation pyrometer with a shielding flange and from a contact thermometer for intermittent correction. The estimated error of the measurement was less than $1 \%$.
\end{abstract}

\section{Introduction}

A continuous annealing process (CAP) has been developed currently to meet the requirements of increasing the productivity, improving the quality of products, and bringing forth new products. Since steel strips run at a high speed through the furnace, accurate information about annealing conditions has to be known quickly to control the process. One of the most important factors to be known is the surface temperature of strip in a continuous annealing furnace (CAF). Accordingly, the establishment of a measuring technique with a good accuracy is strongly requested. Although there have been some reports $^{1,2)}$ on the temperature measurement of steel strips, neither investigations on accuracy nor considerations as a total system for process control have been appeared. Therefore, the present authors have started to develop a new temperature measurement system for cold rolled steel sheets and silicon steel sheets in CAF.

Following two conditions should be taken into consideration in the investigation.

(1) The temperature of steel strip is lower than that of furnace wall or heat source.

(2) The emissivity of steel strip is considerably low in CAF using non-oxidizing atmosphere.

For an on-line application, following two items are most important.

1) To establish the on-line correction method.

2) To maintain the good accuracy of instruments.

This paper describes the results of analyses and applications to the actual process.

\section{Simulations of Temperature Measurement in $C A F$}

\section{Shielding Effects}

In any systems of interchanging radiation among diffusely reflecting surfaces, the effective radiation energy, $G_{i}$, per unit area of surface $i$ is derived as follows.***

$$
\begin{gathered}
G_{i}=\varepsilon_{i} \cdot E_{b}\left(T_{i}\right)+\left(1-\varepsilon_{i}\right) \sum_{k=1}^{n} G_{k} \cdot F_{i k} \\
(i=1,2, \ldots, n)
\end{gathered}
$$

where $\varepsilon_{i}$ and $T_{i}$ are the emissivity and the temperature of surface $i$, respectively. The angle factor, $F_{i k}$, designates the fraction of radiation energy leaving surface $i$ and reaching surface $k . E_{b}\left(T_{i}\right)$ means the blackbody radiation energy at temperature $\mathcal{T}_{i}$. The spectral emissive power is given by Planck's law as follows:

$$
E_{b}\left(T_{i}\right)=\frac{C_{1}}{\lambda^{5}\left[\exp \left(C_{2} / \lambda \cdot T_{i}\right)-1\right]}
$$

where $\lambda$ is wavelength, $\mu \mathrm{m}$,

$$
\begin{array}{ll}
C_{1}=3.7413 \times 10^{4} & \left(\mathrm{~W} \cdot \mathrm{cm}^{-2} \cdot \mu \mathrm{m}^{4}\right) \\
C_{2}=1.4388 \times 10^{4} & (\mu \mathrm{m} \cdot \mathrm{K})
\end{array}
$$

In case of $\lambda \cdot T<3000 \mu \mathrm{m} \cdot \mathrm{K}, \mathrm{Eq} .(2)$ is approximated by Wien's law as below:

$$
E_{b}\left(T_{i}\right)=\frac{C_{1}}{\lambda^{5} \exp \left(C_{2} / \lambda \cdot T_{i}\right)}
$$

In case of narrow spectral bands and narrow temperature range, the following approximation can be made:

$$
E_{b}\left(T_{i}\right)=A \cdot T_{i}^{n}
$$

where,

$$
\begin{aligned}
& n=C_{2} / \lambda \cdot T_{i} \ldots \\
& A=\text { constant }
\end{aligned}
$$

A model was considered as in Fig. 1 to simulate a temperature measurement system in a furnace, where $\varepsilon_{1}, \varepsilon_{2}$ and $\varepsilon_{3}$ are emissivities of steel strip, furnace wall and shielding flange with diameter $2 R$, respectively. $T_{1}, T_{2}$ and $T_{3}$ are the temperatures of the above three bodies. $F_{i k}$ is the function of the normalized distance, $m$.

The apparent temperature indication, $T_{a}$, of the radiation pyrometer can be obtained from $G_{1} / \varepsilon_{1}$ by introducing the above quantitative values into Eq.

* Originally published in Tetsu-to-Hagané, 61 (1975), 2076, in Japanese. English version received August $28,1975$.

** Fundamental Research Laboratories, R \& D Bureau, Nippon Steel Corp. Ida, Nakahara-ku, Kawasaki 211.

*** The derivation of Eq. (1) is based on Ref. 3). 


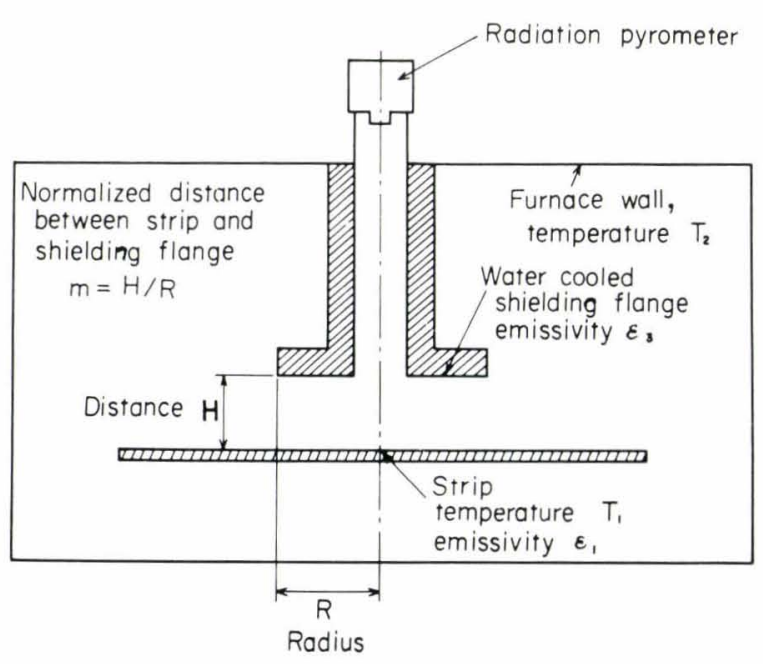

Fig. 1. Schematic diagram of temperature measurement of strip using a pyrometer with shielding flange

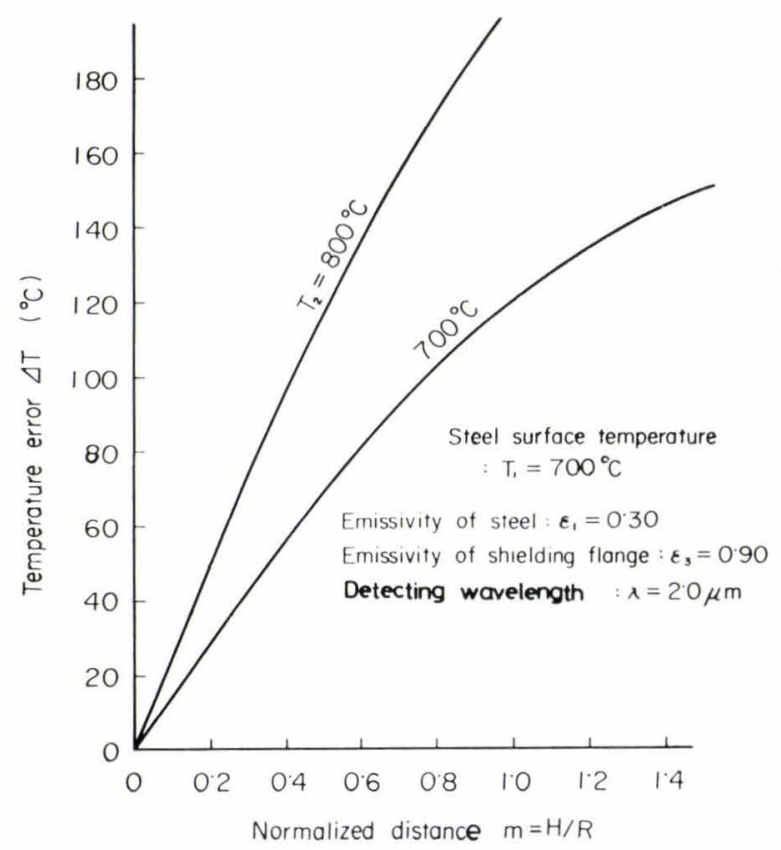

Fig. 2. Relation between $m$ and $\Delta T$ (theoretical)

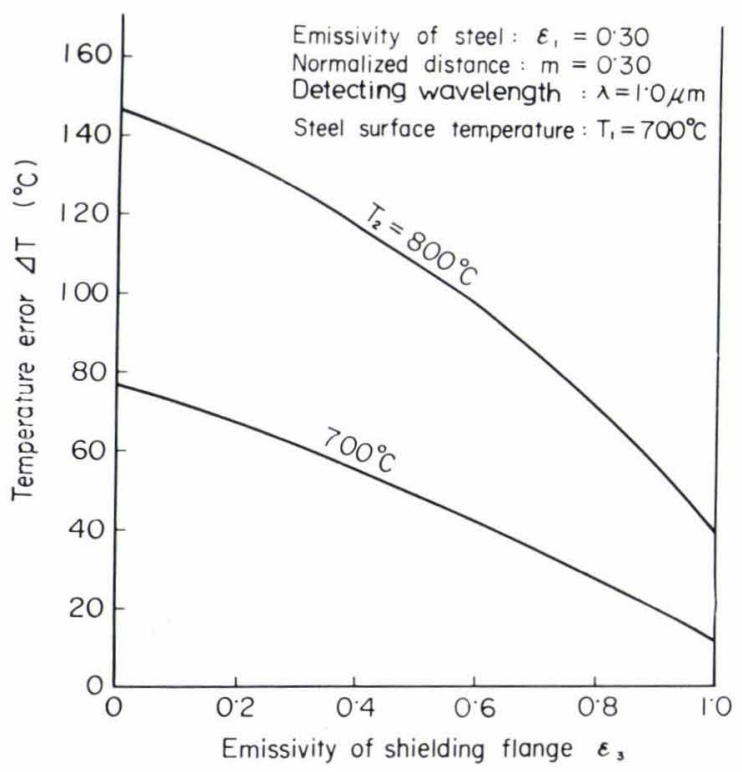

Fig. 3. Relation between $\varepsilon_{3}$ and $\Delta T$ (theoretical)
(1). Some results of computation are discussed below. It is clear in Fig. 2 that the measurement error, $\Delta T$ $\left(=T_{a}-T_{1}\right)$, increases with increasing distance, $m$. $\varepsilon_{3}$ strongly affects $\Delta T$, as seen in Fig. 3, where $\Delta T$ decreases rapidly with increasing $\varepsilon_{3}$. Figure 4 shows that the detecting wavelength, $\lambda$, of the pyrometer also has a strong influence on the temperature measurement in the furnace. That is, $\lambda$ which minimizes $\Delta T$ moves toward the longer wavelength as the difference, $T_{2}-T_{1}$, increases.

From these results, three factors which strongly affect the accuracy are summarized as follows.

i) The normalized distance, $m$

ii) The emissivity of shielding flange, $\varepsilon_{3}$

iii) The detecting wavelength, $\lambda$

These facts are to be confirmed experimentally.

\section{Cooling Effects of Shielding Flange}

The temperature drop of steel strip due to the water-cooled flange could cause the measurement error, and might deteriorate the quality or the shape of strips. This temperature drop was estimated by using a model in Fig. 1. Figure 5 shows an example of the results. It is clear from this figure that the temperature drop is small enough and has no significant effect on strips under the normal operating conditions (line speed $\geq 50 \mathrm{~m} / \mathrm{min}, m \geq 0.2$ ). The results of computation are later confirmed experimentally with on-line measurements (see Figs. 16 and 17).

\section{Fundamental Experiments on Radiation Py- rometry with Shielding Flange}

\section{Measurements on Shielding Effects}

$G_{1}$ in Eq. (1) is rewritten as below:

$$
G_{1}=\varepsilon_{1} \cdot E_{b}\left(T_{1}\right)+\eta \cdot E_{b}\left(T_{2}\right)
$$

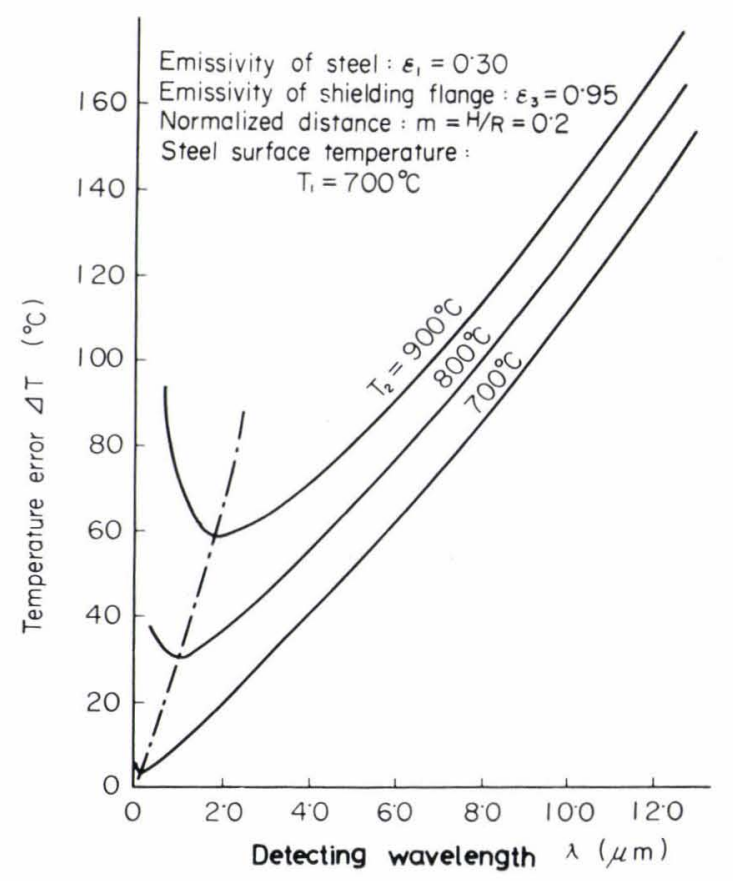

Fig. 4. Relation between detecting wavelength and $\Delta T$ (theoretical) 


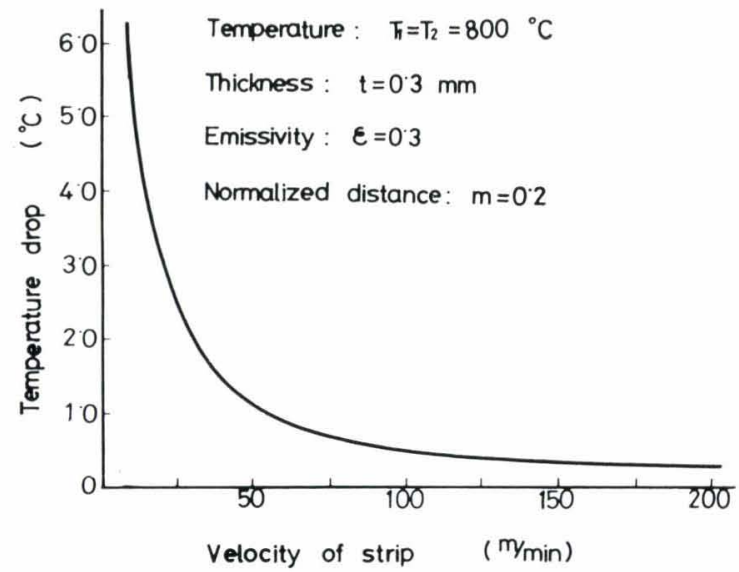

Fig. 5. Strip temperature drop caused by cooling effect of shielding flange (theoretical)

$\eta$ is named as "Back Radiation Factor" in this paper, representing the reflected portion of total radiation energy from furnace walls. Using this $\eta$, the noise energy, $\eta \cdot E_{b}\left(T_{2}\right)$, is given. The output, $E_{b}\left(T_{a}\right)$, of the pyrometer after correction with emissivity, $\varepsilon_{1}$, is given as Eq. (7).

$$
E_{b}\left(T_{a}\right)={ }_{\varepsilon_{1}}^{G_{1}}=E_{b}\left(T_{1}\right)+{ }_{\varepsilon_{1}}^{\eta} \cdot E_{b}\left(T_{2}\right)
$$

where, $T_{a}$ is apparent temperature.

Generally, $\gamma$ is the function of $\varepsilon_{1}, \varepsilon_{3}, m$ and $\lambda$. However, the temperature dependence of $\eta$ can be neglected since steel surface is not oxidized but smooth in the furnace with non-oxidizing atmosphere. In Eq. (6), if $T_{1} \ll T_{2}$, that is, $E_{b}\left(T_{1}\right) \ll E_{b}\left(T_{2}\right)$, $\eta$ is given as below:

$$
\eta=\frac{G_{1}}{E_{b}\left(T_{2}\right)}
$$

The apparatus for the measurement of $\eta$ is shown in Fig. 6. Five CA thermocouples were welded on stainless steel pipe to monitor surface temperatures and the mean was regarded as wall temperature, $T_{2}$. The wall emissivity, $\varepsilon_{2}$, could be regarded as 1.0 because of oxidized surface and multiple reflection effect. Two values were selected for the emissivity of shielding flange, $\varepsilon_{3}$. Namely, $\varepsilon_{3}$ of 0.95 was made by black paint, and 0.5 by brushing it with sandpaper. The surface temperature of the specimen, $T_{1}$, was kept at about $20^{\circ} \mathrm{C}$, so that $E_{b}\left(T_{1}\right)$ could be neglected.

Under the above conditions, both $m$ and $G_{1}$ were simultaneously recorded, keeping $T_{2}$ constant. Then $\eta$ was obtained by using Eq. (8).

\section{Results and Discussion}

Experimental values of $\eta$ are shown in Fig. 7 . From the results of cold rolled steel strips, it was clear that $\eta$ depended on wavelength. In addition, the outstanding difference of $\eta$ was observed between specularly reflecting surface and diffusely reflecting one. Cold rolled steel and brightly annealed stainless steel corresponded to the former, while heavy plate steel and aluminum plate the latter. In our case, specularly reflecting surfaces are desirable. Ap-

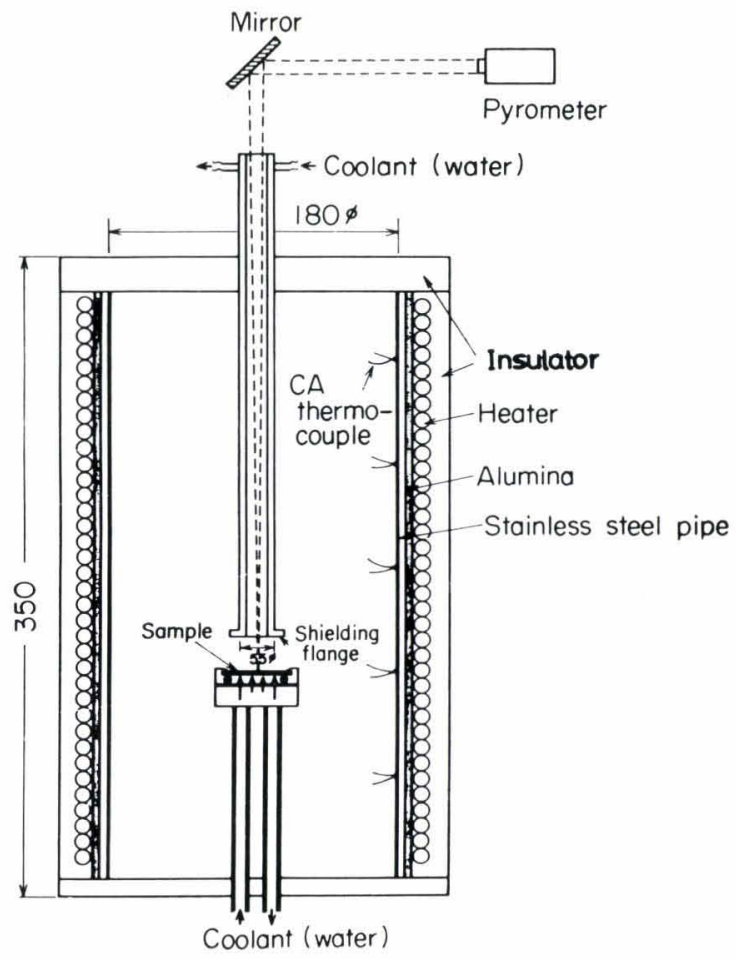

Fig. 6. An experimental apparatus for back radiation factor measurement



Fig. 7. Experimental result of back radiation factor, $\gamma$

Table 1. Experimental values of $\alpha_{\lambda}$

\begin{tabular}{l|c|c|c}
\multirow{2}{*}{\multicolumn{1}{c|}{ Material }} & \multicolumn{3}{|c}{$\alpha_{\lambda}$} \\
\cline { 2 - 4 } & $\lambda=1.0$ & $\lambda=2.0$ & $\lambda=3.0$ \\
\hline Cold rolled steel & 0.024 & 0.020 & 0.009 \\
Silicon steel & 0.025 & 0.021 & 0.009 \\
Stainless steel & 0.013 & 0.005 & 0.004 \\
Heavy plate steel & 0.090 & 0.190 & 0.054 \\
Aluminum & 0.162 & 0.210 & 0.078
\end{tabular}

proximating these curves by the linear relation as,

$$
\eta=\alpha_{\lambda} \cdot m
$$

$\alpha_{\lambda}$ can be defined as $\eta$ at $m=1.0$ and is a measure of specularity for material surface. Table 1 shows $\alpha_{\lambda}$ 
for several materials.

Effects of $\varepsilon_{3}$ are shown in Fig. 8, where the theoretical result from computation in Section $I I$ is also shown. It is remarkable that $\varepsilon_{3}$ has a great influence on the measurement. That is, $\eta$ decreases rapidly with increasing $\varepsilon_{3}$. Therefore, $\varepsilon_{3}$ more than 0.9 is necessary for practical applications. Although the theoretical result has the same tendency as the experimental one, it is quite different quantitatively. This fact also means that cold rolled strip is a specular reflector in the infrared region.

$\eta / \varepsilon_{1}$ in Eq. (7) is a measure of the measurement accuracy. Substituting $\varepsilon_{1}$ values (see Fig. 7) and $\alpha_{\lambda}$ values (see Table 1) of cold rolled steel into Eq. (9), the measure, $\eta / \varepsilon_{1}$, is given as follows:

$$
\begin{array}{lll}
\eta / \varepsilon_{1}=0.069 m & \text { when } & \lambda=1.0 \\
\eta / \varepsilon_{1}=0.091 m & \text { when } & \lambda=2.0 \\
\eta / \varepsilon_{1}=0.060 m & \text { when } & \lambda=8.0
\end{array}
$$

From the above results, as far as $\eta_{/} / \varepsilon_{1}$ is concerned, longer wavelength is desirable, but in fact the conclusion is not so simple. That is, the error, $\Delta T$, might increase with increasing wavelength, since $n$ value in Eq. (5) is small in the region of longer wavelength. ${ }^{2)}$

Equations estimating $J T$ are obtained from either Eqs. (3), (7) and (9) or Eqs. (4), (7) and (9), as below:

$$
\begin{aligned}
\Delta T= & T_{a}-T_{1} \\
= & \ln \left[\begin{array}{c}
\exp \left\{\left(C_{2} / \lambda\right) \cdot\left(1 / T_{1}+1 / T_{2}\right)\right. \\
\exp \left(C_{2} / \lambda \cdot T_{2}\right)+\frac{\alpha_{\lambda} \cdot m}{\varepsilon_{1}} \cdot \exp \left(C_{2} / \lambda \cdot T_{1}\right)
\end{array}\right] \\
& \ldots \ldots \ldots \ldots \ldots \ldots(10)
\end{aligned}
$$

Equation (10) can be used generally and Eq. (11) is used in case of $T_{1} \simeq T_{2} . \Delta T$ is estimated, if $\alpha_{\lambda}$ and $\varepsilon_{1}$ are obtained experimentally, and $T_{1}$ and $T_{2}$ are given according to real conditions in CAF. The error estimation for cold rolled steel is shown in Fig. 9. From this figure it is obvious that in case of $T_{1} \simeq T_{2}$, the wavelength of $1 \mu \mathrm{m}$ is more suitable than $2 \mu \mathrm{m}$ in order to lower $\Delta T$, while in case of $T_{2}-T_{1} \geq$ $200,2 \mu \mathrm{m}$ is better than $1 \mu \mathrm{m}$. In other words, the optimum moves to longer wavelength with increasing difference, $T_{2}-T_{1}$. This result corresponds to theoretical one in Fig. 4 . The allowable distance, $m_{\alpha}$, to keep the error under $\pm 0.5 \%$ is given as follows:

$$
\begin{aligned}
& m_{a}=0.67 \text { when } \lambda=1.0, T_{1}=700^{\circ} \mathrm{C}, T_{2}=800^{\circ} \mathrm{C} \\
& m_{a}=0.50 \text { when } \lambda=2.0, T_{1}=700^{\circ} \mathrm{C}, T_{2}=800^{\circ} \mathrm{C} \\
& m_{a}=0.35 \text { when } \lambda=1.0, T_{1}=700^{\circ} \mathrm{C}, T_{2}=900^{\circ} \mathrm{C} \\
& m_{a}=0.42 \text { when } \lambda=2.0, T_{1}=700^{\circ} \mathrm{C}, T_{2}=900^{\circ} \mathrm{C}
\end{aligned}
$$

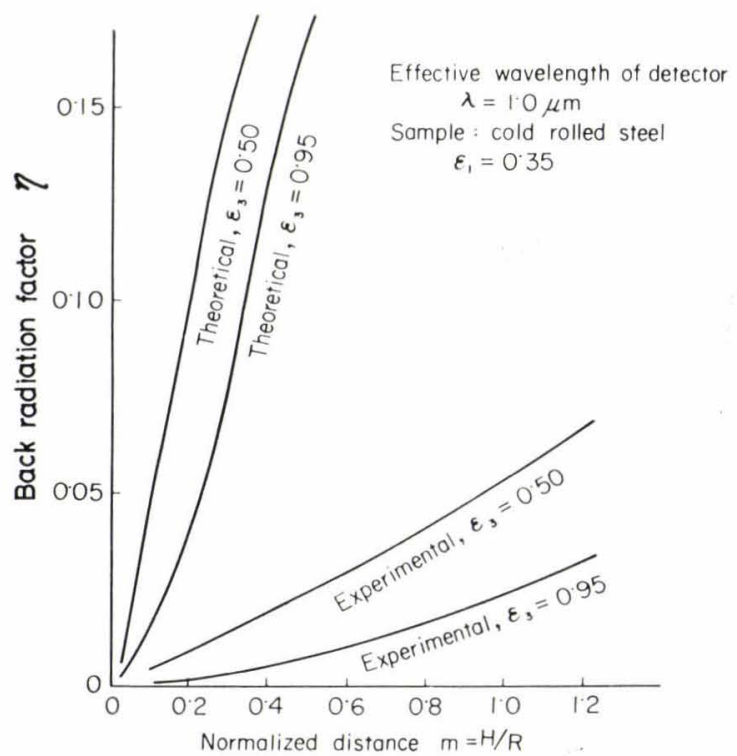

Fig. 8. Effect of $\varepsilon_{3}$ on $\eta$

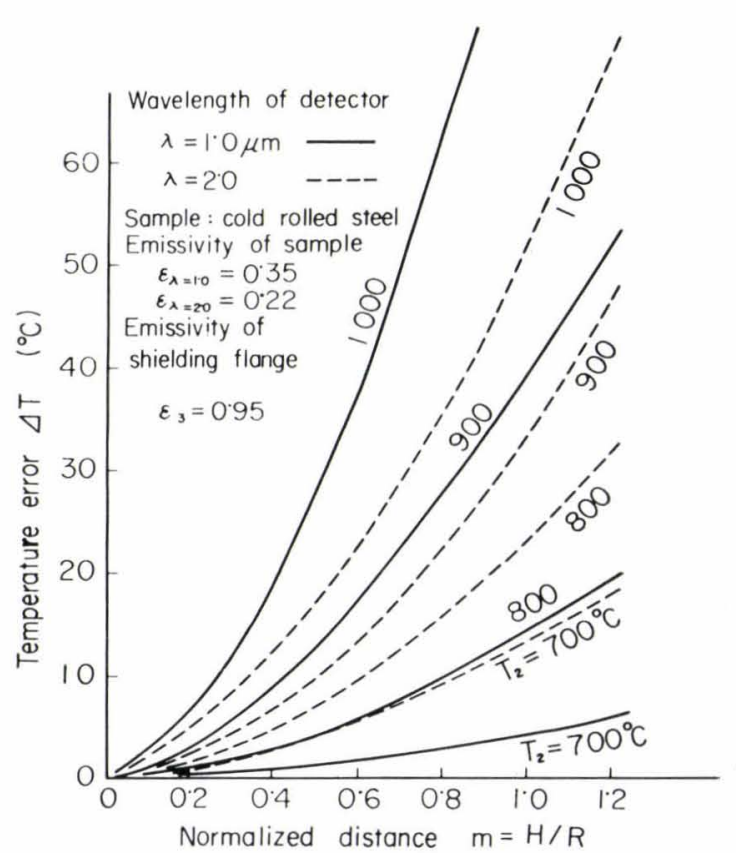

Fig. 9. Relation between $m$ and $\Delta T$ in case of cold rolled steel strip. $\Delta T=T_{a}-T_{1} . \quad T_{a}$ is apparent temperature indicated by a pyrometer. $T_{1}$ is surface temperature of the strip $\left(=700^{\circ} \mathrm{C}\right)$ and $T_{2}$ is wall temperature.

As a consequence, the measurement error could be kept under $\pm 0.5 \%$ in case of $T_{2}-T_{1} \leq 200$ and $m \leq$ 0.35 .

\section{Emissivity Measurements}

\section{Apparatus and Experimental Procedure}

After the previous calibration of the pyrometer with a blackbody furnace, a number of emissivity measurements were made with an apparatus shown in Photo. 1. Mixed gases of $\mathrm{H}_{2}$ and $\mathrm{N}_{2}$ of the same composition as in CAF were constantly supplied into the apparatus. The apparatus was water-cooled to prevent emịssion from itself and the inside wall was 


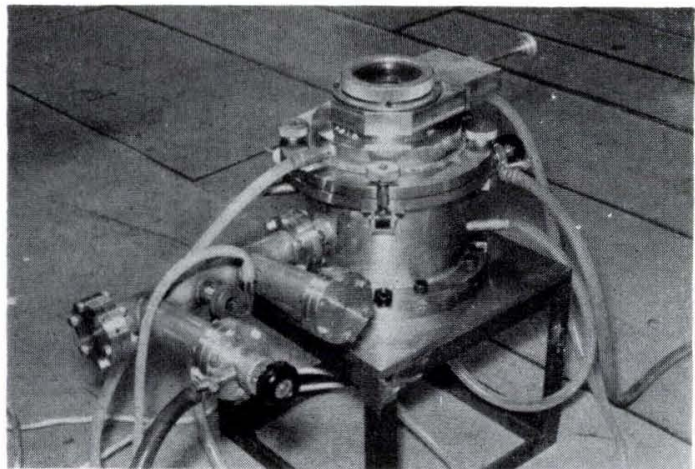

Photo. 1. Emissivity measurement apparatus

coated with black paint to absorb radiation. In this apparatus, disk-type specimen $(60 \mathrm{~mm} \phi)$ was set on a small heater. The temperature, $T$, of the specimen was measured by a CA thermocouple $(0.2 \mathrm{~mm} \phi)$ welded on the surface. The measurement error of the thermocouple had been corrected previously and the specimen could be heated up to $1000^{\circ} \mathrm{C}$. The radiation energy, $E(T)$, from specimen was detected through a window made of fused silica which was set just above the specimen. The transparency of the window was $93 \%$ for the two used pyrometers, therefore the emissivity, $\varepsilon(T)$, was written as follows:

$$
\varepsilon(T)=\begin{gathered}
E(T) \\
0.93 E_{b}(T)
\end{gathered}
$$

Spectral emissivities were measured by modifying a commercial monochrometer, whose wavelength region was from 0.5 to $15 \mu \mathrm{m}$. For this measurement, both the above heater for the specimen and a small blackbody furnace were used and emission from both was compared.

\section{Results and Discussion}

Spectral emissivities of cold rolled steel and silicon steel are given in Fig. 10. The result shows that emissivities are high in visible wavelength region and decrease rapidly within near-infrared region and in case of more than $5 \mu \mathrm{m}$ they are relatively constant. According to Hagen-Rubens' equation, ${ }^{4,5)}$ the emissivity of a smooth metallic surface, $\varepsilon_{\lambda}$, is as follows:

$$
\varepsilon_{\lambda}=36.05 \sqrt{\frac{\rho}{\lambda}}
$$

where, $\rho:$ specific resistivity $(\Omega$-cm)

$\lambda$ : wavelength $(\mu \mathrm{m})$

The result in Fig. 10 has the same tendency about wavelength as Eq. (13), although specimens do not have completely smooth surfaces. Figure 11 shows the dependence of emissivity upon temperature for a constant wavelength. Emissivities increase slightly with temperature. Since $\rho$ is proportional to the temperature $T$ at higher range, ${ }^{6)} \mathrm{Eq}$. (13) may become Eq. (14).

$$
\varepsilon_{\lambda} \propto \sqrt{ } T
$$

The result in Fig. 11 can be explained with Eq. (14). Accordingly, the pyrometer emissivity setting must



Fig. 10. Result of spectral emissivity measurement in case of cold rolled steel and silicon steel

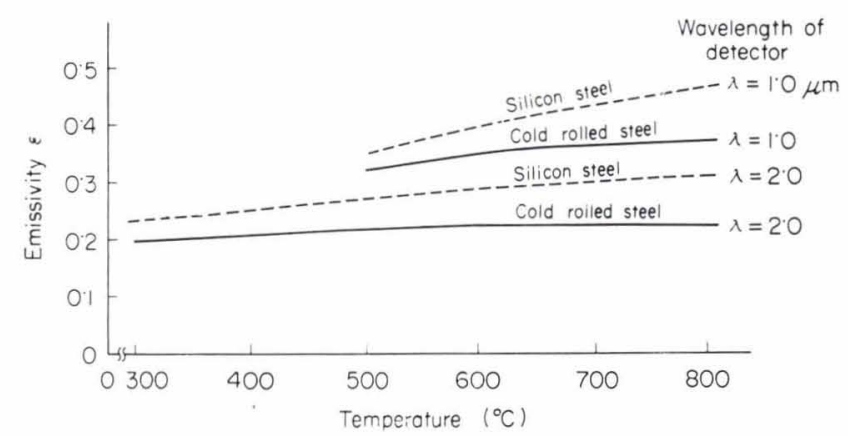

Fig. 11. Relation between emissivity and temperature in case of cold rolled steel and silicon steel



Fig. 12. Relation between emissivity and silicon content in case of silicon steel

be adjusted to correspond to the zones of CAF. Figure 12 denotes the dependence of emissivity upon silicon content for a constant wavelength. It can be clearly seen that the higher silicon content, the higher emissivity under $3 \%$, beyond which emissivity becomes constant. $\varepsilon_{\lambda}$ in Eq. (13) increases with increasing electrical resistivity, which increases with increasing silicon content. Therefore, the result in Fig. 12 corresponds to Eq. (13). So, the pyrometer emissivity setting has to be adjusted according to silicon content of steel. Carbon content or other elements did not influence emissivities of both cold rolled steel and silicon steel. For on-line measurement, the stability of emissivity is the most important of all. Laboratory experiments showed good stable results as seen in Fig. 13. The emissivity change due to quality or surface conditions (dull or bright) was very small in case of cold rolled steel. The average, $\bar{\varepsilon}$, was 0.22 and the standard deviation, $\sigma$, was 0,006 by using 


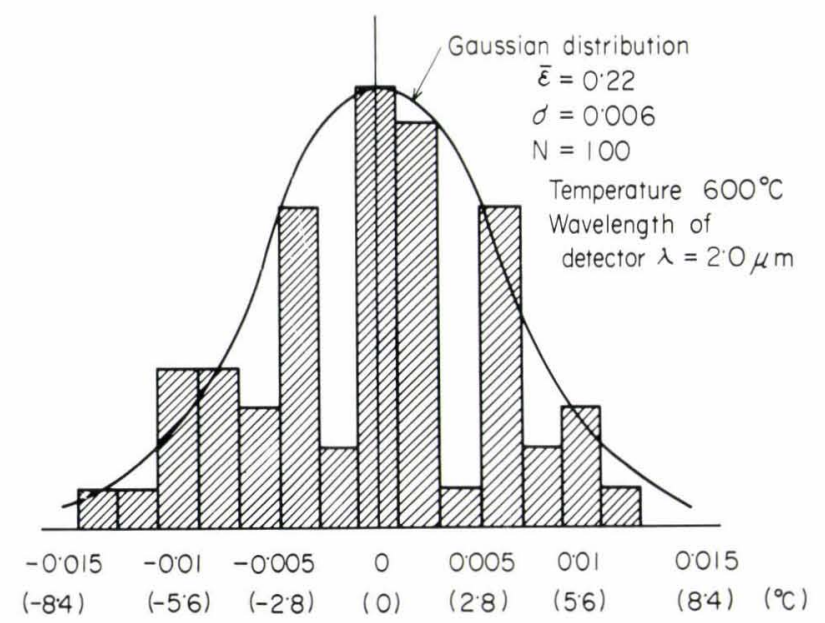

Fig. 13. Fluctuation of emissivities of cold rolled steels. Values in parentheses are equivalent errors at $600^{\circ} \mathrm{C}$.

$\mathrm{PbS}$ cell detector. This result corresponded to the error of about $3^{\circ} \mathrm{C}$ at $600^{\circ} \mathrm{C}$.

The optimum detecting wavelength of a pyrometer for on-line use could be selected as $2 \mu \mathrm{m}$ by substituting experimental values obtained in Sections $I I I$ and $I V$ into Eq. (10). Therefore, the authors adopted a $\mathrm{PbS}$ cell (detecting wavelength is $2 \mu \mathrm{m}$ ).

\section{Development of Correction Techniques}

\section{Contact-type Thermometer}

If this method of temperature measurement is to be extended to on-line use, some factors must be considered, which cannot always be fully realized in the laboratory, such as gas composition, catenary of strip, and localization of heat sources in a real furnace. These factors may differ in each furnace and so it is very important to correct the measurement technique somehow to ensure the good accuracy. Therefore, a contact-type thermometer has been developed by the authors for intermittent checking.

The correction method is a simultaneous measurement of strip temperature with both the pyrometer and the contact-type thermometer. This thermometer was provided with a thin stainless "guard" sheet ( $40 \mathrm{~mm}$ long, $16 \mathrm{~mm}$ wide and $0.2 \mathrm{~mm}$ thick) on which a CA thermocouple was welded. The guard sheet was used to protect the thermocouple from wear and to maintain a good heat contact with steel strip. ${ }^{7)}$ Figure 14 shows the cross section of the contact-type thermometer used in the actual process. In order to improve the accuracy, the guard sheet was insulated and has a spring mechanism which followed the flapping of steel strip. However, a slight scratch on strip surface was unavoidable due to the contact. In addition, this thermometer was water-cooled to be effective even in a furnace. The detecting portion including the guard sheet was renewed after 24 hours' service to avoid the error due to the degeneration of the thermocouple in a high temperature furnace.

The resultant accuracy was within the error of $\pm 5^{\circ} \mathrm{C}$ at strip temperature of $800^{\circ} \mathrm{C}$. The detail

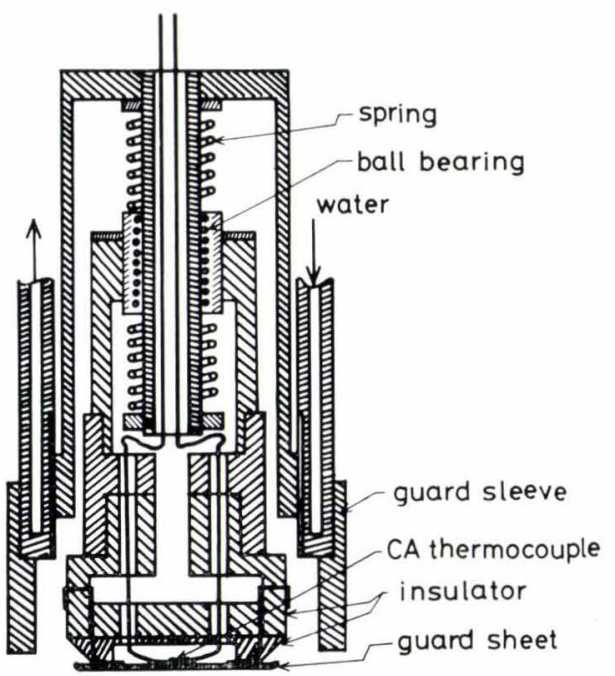

Fig. 14. Cross section of a contact-type thermometer

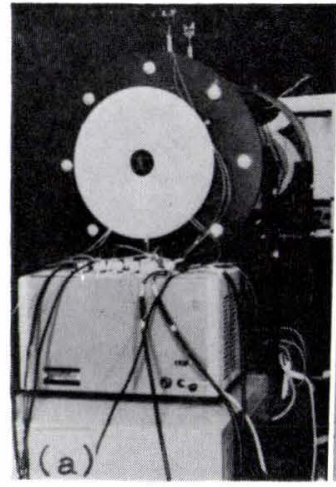

(a) High precision type

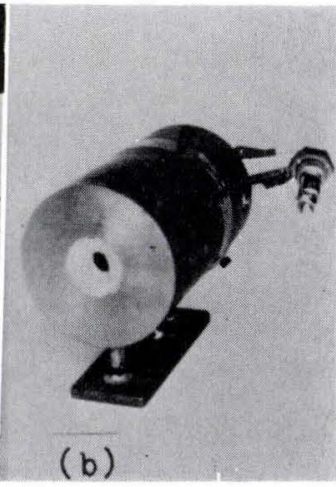

(b) Simplified type
Photo. 2. Blackbody furnaces for pyrometer calibration

investigations on error factors are omitted in this paper.

\section{Simplified Blackbody Furnace}

It is very important to carry out the calibration as simply and quickly as possible on production line. Therefore, the authors also developed a simplified portable blackbody of $10 \mathrm{~mm} \phi$ aperture and $60 \mathrm{~mm}$ length. The effective emissivity of this blackbody was 0.98. The temperature of furnace was measured by a CA sheath thermocouple buried in the bottom of the blackbody and was controlled by a PID controller. The covering temperature range was $50^{\circ}$ to $1000^{\circ} \mathrm{C}$ and the raising time up to $1000^{\circ} \mathrm{C}$ was about $10 \mathrm{~min}$. The calibration of this blackbody was done by using a high precision blackbody furnace in our laboratories, the effective emissivity of which was 0.997. Consequently, the calibration on production line was greatly simplified and the accuracy of on-line measurements was improved because all-round maintenance of the system became possible. Photograph 2(a) shows a high precision blackbody furnace and Photo. 2(b) a simplified one.

\section{Measurements on Production Line}

1. Measurement System

A configuration of the developed system is shown 
in Fig. 15. A water-cooled shielding flange set above steel strip is movable vertically. A continuously measuring pyrometer is placed outside of the furnace through an optical filter. This filter is purged by passing $\mathrm{N}_{2}$ gas to prevent dimming and can be renewed if necessary. On the other hand, an intermittently measuring contact-type thermometer is set under steel strip. It is housed outside of the furnace normally.

\section{On-line Measurements}

Figure 16 shows the comparison between emissivity readings measured in the laboratory and on production line. Results on production line using the contact-type thermometer were coincident with laboratory results, hence the accuracy of the system was ensured. Figure 17 gives the relation between back radiation noise and distance, $m$. The line speed $(150 \mathrm{~m} / \mathrm{min})$ was sufficiently high, so that the temperature of strip $(0.8 \mathrm{~mm}$ thick) was not affected by the flange. The back radiation noise increased about $5^{\circ} \mathrm{C}$, as the distance, $m$, changed from 0.1 to 1.0 . Figure 18 shows the same relation as in Fig. 17, but the strip was cooled to some extent because the line speed was comparatively slow $(40 \mathrm{~m} / \mathrm{min})$ and the strip was thinner $(0.35 \mathrm{~mm})$. The contact-type thermometer indicated temperature decrease of about $4^{\circ} \mathrm{C}$ with the change of $m$ from 0.1 to 1.0 . The pyrometer,

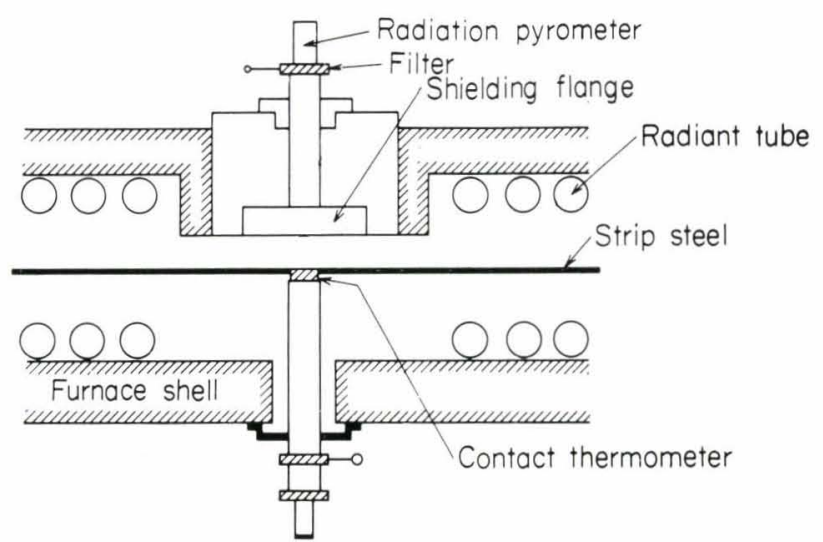

Fig. 15. Configuration of a temperature measurement system

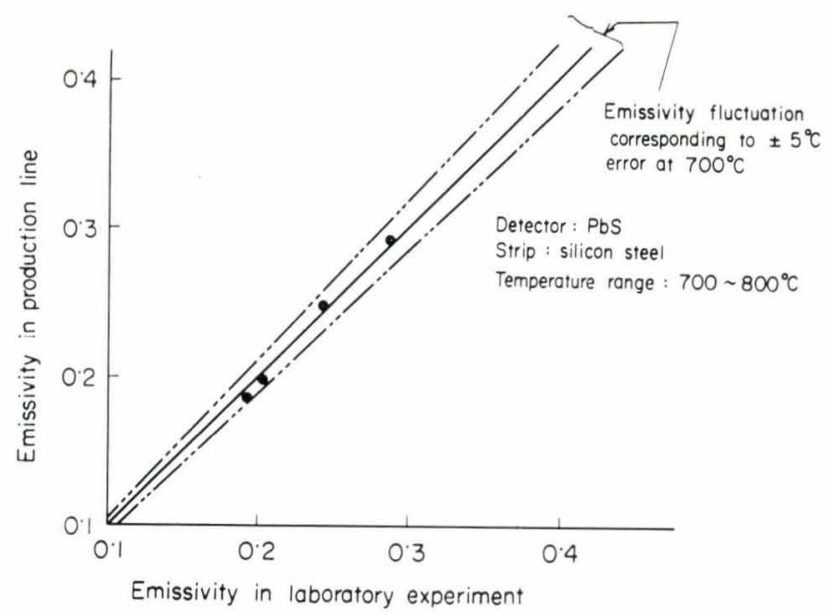

Fig. 16. Comparison of emissivities between the production line and the laboratory however, indicated the change of about $18^{\circ} \mathrm{C}$. These results mean that the back radiation noise was about $14^{\circ} \mathrm{C}$ (this corresponds to the result of Fig. 9, see solid line of $T_{2}=800^{\circ} \mathrm{C}$ ) and that at $m=0.1$ the strip was cooled by $4^{\circ} \mathrm{C}$. As shown in Fig. 19, the pyrometer indications followed the strip temperature accurately. The temperature change of $32^{\circ} \mathrm{C}$ on the pyrometer

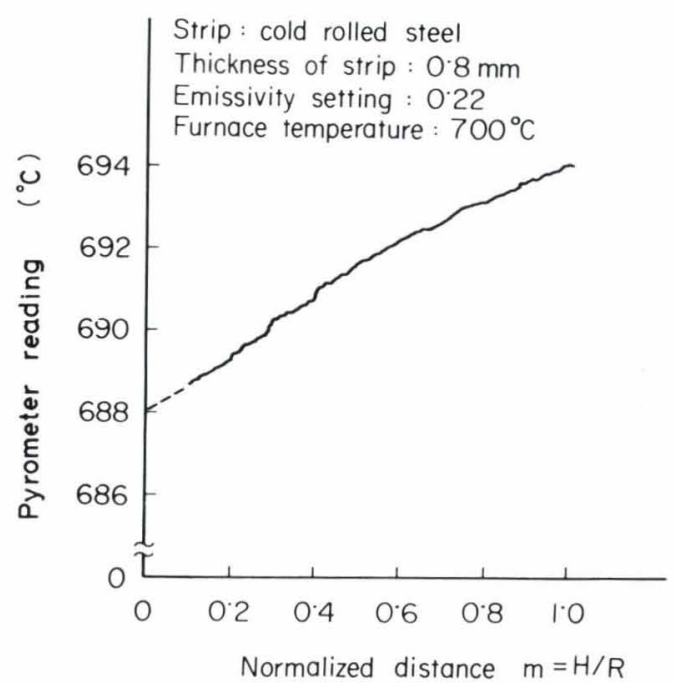

Fig. 17. Pyrometer reading vs. distance reading. Back radiation noise decreases as shielding flange approaches to strip.

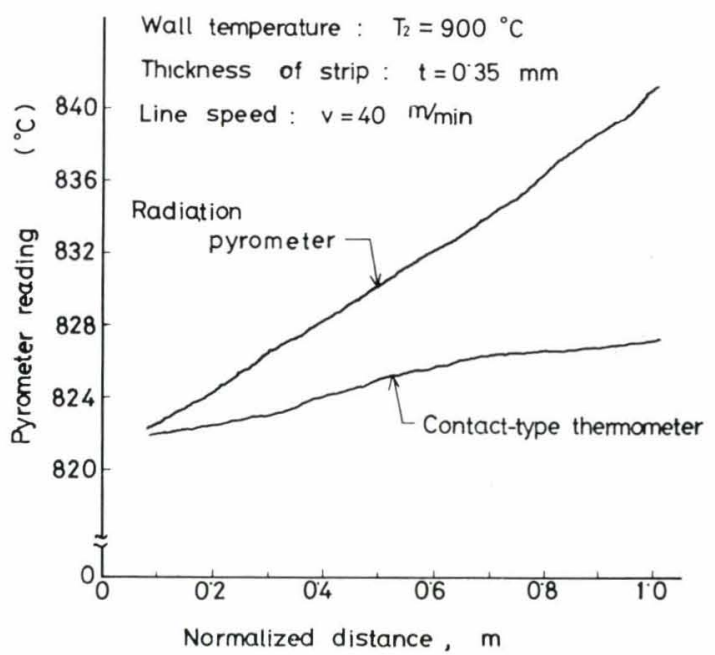

Fig. 18. Pyrometer reading vs. distance, $m$. The strip is cooled to some extent by shielding flange because of low speed and thin strip.

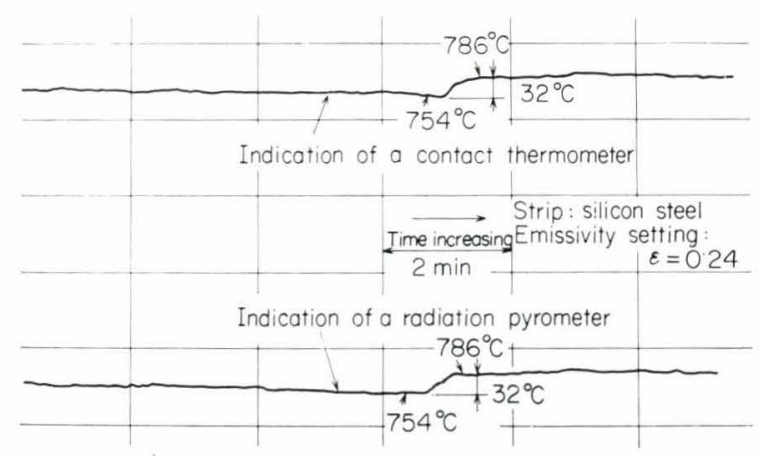

Fig. 19. Correspondence between pyrometer indication and contact-type thermometer indication 
reading corresponded exactly to that on the contacttype thermometer reading. Figure 20 shows examples of measurements, such as temperature change due to the change of line speed, detection of a welded portion and effects of cooling zone. A cooler blowing cooled gases onto strip surface reduced the temperature rapidly. Conversely, when the cooler was turned off, the temperature rose slowly. These results show that the cooler can be an imporatnt manipulator for process control. ${ }^{8)}$ Welded portions generally has rough surfaces and their emissivities were higher than usual, hence the pyrometer often indicated a higher temperature than normal like a pulsed shape. Accordingly, the temperature readings were meaningless, but they represented the signals of the welded portions. Change of line speed is the most effective variable in controlling strip temperature. Increasing the speed, the temperature dropped in heating, soaking or overaging zones, but rose in cooling zone. Figure 21 gives an example of simultaneous measurements during operation of CAF having five zones. The measured values are considered to be indispensable process parameters for the controls of respective zones and the processing system on the whole.

\section{Conclusions}

The following conclusions were drawn based on the results of investigations.

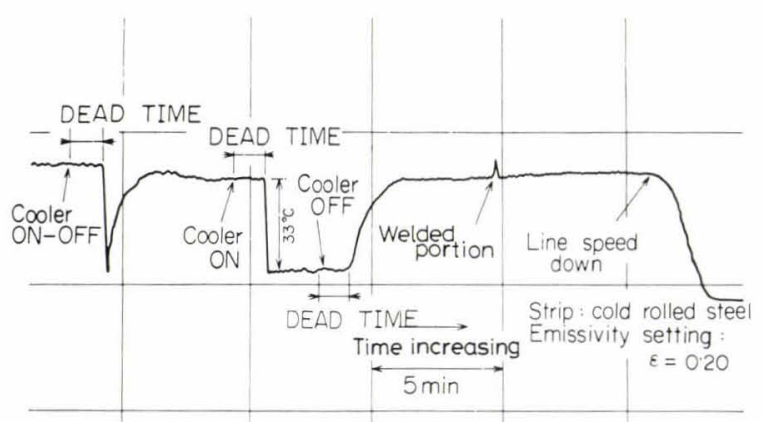

Fig. 20. Various measurement examples in cooling zone
(1) The emissivities of steel strips were stable in a continuous annealing furnace with inert or reducing gases, and the radiation pyrometry was sufficiently accurate.

(2) The emissivity characteristics were clarified. They depended on specification and temperature of steel, and on detecting wavelength.

(3) The stray radiation energy from furnace walls or heat sources (back radiation energy) was quantitatively examined. This energy depended on the shape and surface conditions of shielding flange, the distance between strip and flange, detecting wavelength and finally temperatures of furnace wall and strip.

On the basis of the above conclusions, the accuracy of measurements was estimated by the quantitative analysis. Then the system was designed and constructed from a radiation pyrometer with shielding flange and from a contact-type thermometer for intermittent correction. As a result, the temperature measurement became possible within an estimated error of $1 \%$.

This system has been applied for the Continuous Annealing and Processing Line (CAPL) for cold rolled steel strips at Kimitsu Works of Nippon Steel Corp. and has been operated in many other processes satisfactorily since 1972, increasing the productivity as well as the quality, and moreover generating useful information for the development of another new process.

\section{Nomenclature}

$A: \quad$ constant $\left(\mathrm{W} \cdot \mathrm{cm}^{-2} \cdot \mu \mathrm{m}^{-1} \cdot \mathrm{K}^{-n}\right)$

$C_{1}$ : Planck's first constant $\left(=3.7413 \times 10^{4}\right)$ $\left(\mathrm{W} \cdot \mathrm{cm}^{-2} \cdot \mu \mathrm{m}^{4}\right)$

$C_{2}$ : Planck's second constant $\left(=1.4388 \times 10^{4}\right)$ $(\mu \mathrm{m} \cdot \mathrm{K})$

$E_{b}\left(T_{i}\right)$ : blackbody radiation energy at temperature $T_{i}\left(\mathrm{~W} \cdot \mathrm{cm}^{-2} \cdot \mu \mathrm{m}^{-1}\right)$

$F_{i k}$ : angle factor, fraction of radiation energy leaving surface $i$ and reaching surface $k(-)$

$G_{i}$ : effective radiation energy per unit area of


Fig. 21. Temperature measurements during operation at a continuous annealing furnace having five zones 
surface $i\left(\mathbf{W} \cdot \mathrm{cm}^{-2} \cdot \mu \mathrm{m}^{-1}\right)$

$G_{1}$ : effective radiation energy per unit area of measured strip $\left(\mathrm{W} \cdot \mathrm{cm}^{-2} \cdot \mu \mathrm{m}^{-1}\right)$

$H$ : distance between measured strip and shielding flange $(\mathrm{m})$

$m$ : normalized distance between measured strip and shielding flange $(=H / R)(-)$

$m_{a}$ : allowable normalized distance to keep temperature measurement error under $\pm 0.5 \%$ $(-)$

$n: \quad C_{2} / \lambda \cdot T_{i}(-)$

$R$ : radius of shielding flange $(\mathrm{m})$

$T_{1}$ : temperature of measured strip $\left({ }^{\circ} \mathrm{C}, \mathrm{K}\right)$

$T_{2}$ : temperature of furnace wall $\left({ }^{\circ} \mathrm{C}, \mathrm{K}\right)$

$T_{3}$ : temperature of shielding flange $\left({ }^{\circ} \mathrm{C}, \mathrm{K}\right)$

$\mathcal{T}_{a}$ : apparent temperature indicated by radiation pyrometer $\left({ }^{\circ} \mathrm{C}, \mathrm{K}\right.$ )

$\alpha_{\lambda}$ : measure of specularity for material surface $(\eta / m)(-)$

$\Delta T$ : temperature measurement error $\left(=T_{a}-\right.$ $\left.T_{1}\right)\left({ }^{\circ} \mathrm{C}, \mathrm{K}\right)$

$\eta$ : "Back Radiation Factor", which represents reflected portion of total radiation energy from furnace wall $(-)$

$\lambda$ : detecting wavelength $(\mu \mathrm{m})$

$\varepsilon_{\lambda}$ : spectral emissivity of wavelength $\lambda(-)$ $\varepsilon_{1}$ : emissivity of measured strip (-)

$\varepsilon_{2}$ : emissivity of furnace wall $(-)$

$\varepsilon_{3}$ : emissivity of shielding flange $(-)$

$\rho: \quad$ specific resistivity $(\Omega-\mathrm{cm})$

\section{Acknowledgements}

The authors would like to thank Mr. K. Watanabe and Mr. S. Murashima in Instrumentation Group of Plant Engineering \& Technology Center and Mr. M. Tokita and Mr. H. Karashima in Designing Dept. of Plant \& Machinery Div. in Nippon Steel Corp. for their close cooperation.

\section{REFERENCES}

1) R. Barber: Industrial and Process Heating, (1967), Feb.

2) T. P. Murray: Iron Steel Eng., 48 (1971), 33.

3) E. M. Sparrow and R. D. Cess: Radiation Heat Transfer, Brooks/Cole Publ., (1967), 77.

4) N. F. Mott and H. Jones: The Theory of the Properties of Metals and Alloys, Dover Publ., (1958), 105.

5) N. W. Snyder: Trans. ASME, 76 (1954), 541.

6) C. Kittel: Introduction to Solid State Physics, John Wiley \& Sons, (1966), 3rd ed., Chap. 7.

7) M. Yokob: Heat Transfer, II, John Wiley \& Sons, (1965), 151.

8) J. Ohno and T. Iuchi: Japanese Patent Appli. No.4928512. 